\title{
An optimization strategy of thermal power unit based on nonlinear and fuzzy control of coordinated control system
}

\author{
Haoxun Shi ${ }^{1, a}$ \\ ${ }^{1}$ Zhejiang Energy Group R\&D, Hangzhou 310003, China. \\ a1203105280@qq.com
}

Keywords: Severe peak load regulation, coordination and optimization, nonlinear PID, fuzzy control.

\begin{abstract}
The study proposes optimization strategies which based on nonlinear and fuzzy control of coordinated control system of thermal power unit. On the basis of direct energy balance(DEB) method, variable gain and nonlinear course are introduced to improve features of control system and PID parameters are adjusted using fuzzy algorithms under real-time circumstances. The scheme was applied to and optimized the coordinated control system in a power grid, which significantly enhances power adjusting speed and the ability of severe peak load regulation.
\end{abstract}

\section{Introduction}

With the extraordinary development of wind, photovoltaic and other new energy, affected by random, intermittent, the anti-regulatory, large fluctuation and other factors, the pressure of regulating measures and ability in load adjustment is notably increased. while the structure of power source that mainly depends on thermal power ,hydropower, pumped storage, gas, electricity and other quick adjusting power supply shortage in our country made the frequency and peak modulation face great difficulties. Gansu grid, for example, as of February 2013, Gansu province's power generation capacity has reached 30.1207 million kilowatts, of which thermal power installed capacity of 15.508 million kilowatts, accounting for 51.49 percent, hydropower installed capacity of 7,356,100 kilowatts, accounting for 24.4 percent, wind power and solar energy installed capacity 7256600 kilowatts, accounting for $24.1 \%$ [1]. Thermal power unit must play the roles of frequency and peak modulation after the new energy are accessed.

The task of thermal power unit coordinated control system is to make the unit as quickly as possible in response to changes in Power load, meet the requirements of power adjusting speed and the ability of severe peak load regulation, and should try to ensure that the main steam pressure is stable. In order to enhance the ability of frequency and peak modulation, on the basis of direct energy balance(DEB) method, variable gain and nonlinear course are introduced to improve features of control system, PID parameters are adjusted using fuzzy algorithms under real-time circumstances, power adjusting speed is enhanced greatly and the unit interval of stable operation is expanded, which enhances the ability of frequency and peak modulation.

\section{Coordinated control system analysis}

The effects of regenerative coefficient $\mathbf{C}_{\mathbf{b}}$ on the control quality. In coordinated control system, the feedback signal of the energy controller is heat signal, forms a dynamic and static equilibrium with boiler load demand instruction [2].

$$
P+C_{b} \frac{d P_{d}}{d t}
$$

In formula (1), $C_{b}$ means the regenerative coefficient of boilers, to some extent $C_{b}$ underestimates heat storage capacity of the boiler when $\mathrm{C}_{\mathrm{b}}$ is a little smaller, resulting in fuel adjustment appeared overshoot, or even cause instability in the control system; while it means the excess of the actual boiler storing capability when $C_{b}$ is a litter larger, which will have a significant impact on system similarly [5]. 
The effects of energy directive differential gain on the control quality.Boiler energy directive dynamic correction circuit can be described with Equation (2) :

$$
Y_{\text {out }}(s)=\frac{a s}{b s+1} y_{n}(s)
$$

Among them, differential gain is a b, inertia time constant is b. B generally has little effect on the control quality, but the differential gain has a greater impact on the control quality, easy to cause system instability.

The effects of the tone hysteresis character on the control quality.The steam turbine damper has more obvious hysteresis characteristics, In load adjusting circuit, If the integral gain link function is stronger, will cause the load oscillation [3], not stable near a set value, serious impact on system performance and even instability.

\section{The DEB control strategy optimization}

As a control strategy, direct energy balance method is used most widely in unit coordinated control system. DEB energy controller Settings is energy demand signal, feedback value is heat signal:

$$
\begin{gathered}
N R G D=\left(P_{s} \times P_{1} / P_{T}\right) \times\left[1+K \times d\left(P_{s} \times P_{1} / P_{T}\right) / d t\right. \\
H R=P_{1}+C_{b} \times d P_{D} / d t
\end{gathered}
$$

Because it will make pressure fuel is simplified to single stage control from cascade control, and can maintain machine's pressure before is equal to the given value, the integral correction, no need to apply pressure ,compared with the traditional coordinated control system, the parameter sets easier, system is simple and practical.

Limiting filtering ALF method.DEB control energy demand instruction medium voltage is stronger than the noise of signal, which will enlarge further after differential link compensation, we must carry out effective filtering [4].Usually adopted inertia filtering method can effectively overcome the noise, but at the expense of signal sensitivity at the same time. Limiting filtering ALF rules can effectively filter the noise, and do not form a delay signal.

The design principles of limiting filtering ALF method is setting a signal average amplitude as filtering first, forming a noise filtering area, so it can effectively overcome the noise inside the filter with amplitude, and make the useful signal amplitude variation greater than filtering to obtain true reflection. Energy instructions' form as shown in the figure below:

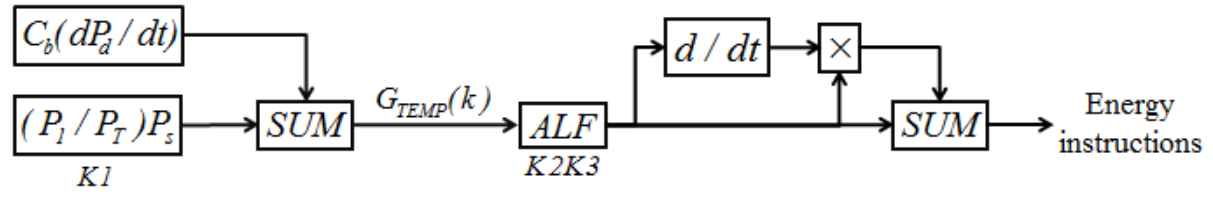

Fig. 1 Energy instructions' form

In "Fig.1", K1 is adjusting pressure P1 scale factor; K2 is bandwidth of ALF; K3 is filter time constant of ALF; K4 is time constant of differential Link; K5 is inertial time constant.

The described formula of ALF is as follows:

$$
A_{1}(k)=G_{\text {TEMP }}(k)-G_{\text {TEMP }}(k-1)+\frac{K_{3}}{K_{3}+T_{C}} A_{1}(k-1)
$$

The schematic diagram is as follows:

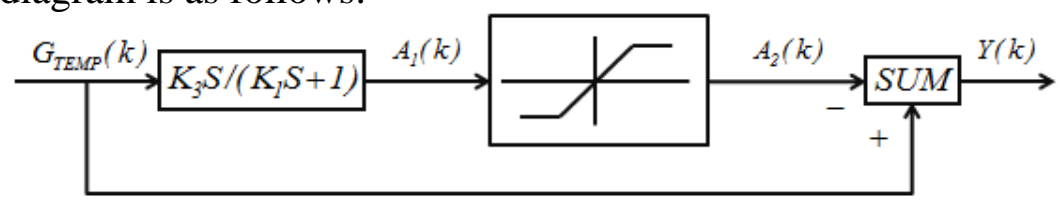

Fig. 2 ALF's schematic diagram

For nonlinear saturation in the figure, when limit amplitude $\mathrm{K} 2$ is large, the output $\mathrm{Y}(\mathrm{k})$ is the output of input signal GTEMP (k) passing through the filter of one-order inertial ; When the K2 is small, ALF output hardly processed. When K2 setting more accurately, it not only can overcome the power frequency interference of signal measurement, and can also make disturbance signal through 
that reflects the change speed enough during the load change, so that the turbine changes of the operation condition get correct reflection.

The use of nonlinear PID. With PID control algorithm's simplicity, good robustness and high reliability, it is widely used in process control, but when the control object has large inertia, nonlinear, strong coupling and strong interference, the contradiction between quickness and overshoot is difficult to solve [5], nonlinear PID can effectively solve the contradiction. Nonlinear PID real-time generates the function of adjustable parameters through the size of the system output error , so can obtain nonlinear PID available model, in which the proportional gain is the mathematical model the constant and deviation is multiplied by the square.

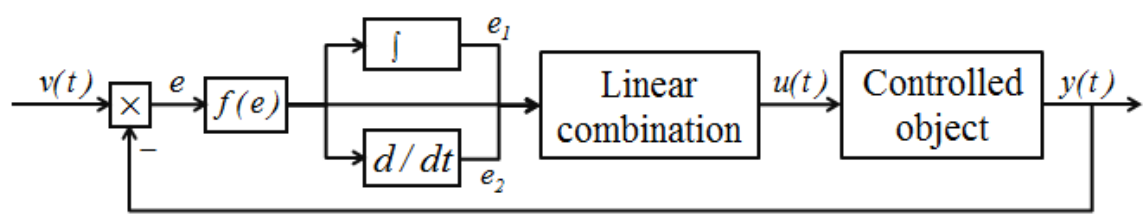

Fig. 3 The nonlinear error PID

The design of Nonlinear dynamic compensation. The use of nonlinear PID will significantly improve control quality of main steam pressure in steady operating conditions. But the quality of the dynamic conditions to improve needs the use of nonlinear dynamic compensation. In the process of load change, need establish different acceleration signal, such as air flow, fuel, for the correction of the dynamic response process .In addition, in order to timely supplement boiler heat storage, major auxiliary control system parameters must be properly overshoot, and other parameters also have corresponding overshoot. Through the excessive adjustment of the boiler input instructions in the process of rising changeable load and the lacking adjustment of it in the process of declining changeable load and accelerating the control process of unit combustion, improves the controllability of the main steam pressure.

Nonlinear dynamic compensation link adopts intelligent judgment, to determine the dynamic compensation signal amplitude, compensation start time, duration and end time, etc. The main principle is as follows:

(1)The compensation signal amplitude is determined according to the load change rate, the width of load change and the target load instructions and other related quantities. The faster the rate of change, the more wide the width, the higher the target load instruction, the greater the dynamic compensation range, and vice versa. But in the unit load up high and close to the fuel quantity range or load down close to the low fuel quantity limit should be treated differently.

(2)The compensating signal duration depends on deviation between the load actual instruction, target load with actual load after the unit limiting rate , and so on. When the actual load instruction is close to target load or the actual load close to the target load, the amount of compensation come to zero.

(3)When the load instruction then change reversely after the change, the dynamic compensation immediately end, trigger backward overshoot at the same time.

(4)The variable load, increase rate, decline rate, investment time, removal time of the amount of overshoot can adjust flexibly according to the change of load, pressure, steam temperature when changing load.

(5)Limiting compensation link rate according to the width of changeable load.

The application of fuzzy rule table.Steady variable integral circuit need to make a fuzzy self-tuning on the strength of the integral action. The self-tuning process controlled by complex rules, that is establishing fuzzy control rule table. Grade of fuzzy control rules table is associated with its control precision, thus can through traditional function block, addition block, switch block and algorithm blocks to achieve any level of fuzzy control rules table. Relevant schematic diagram is as follows: 

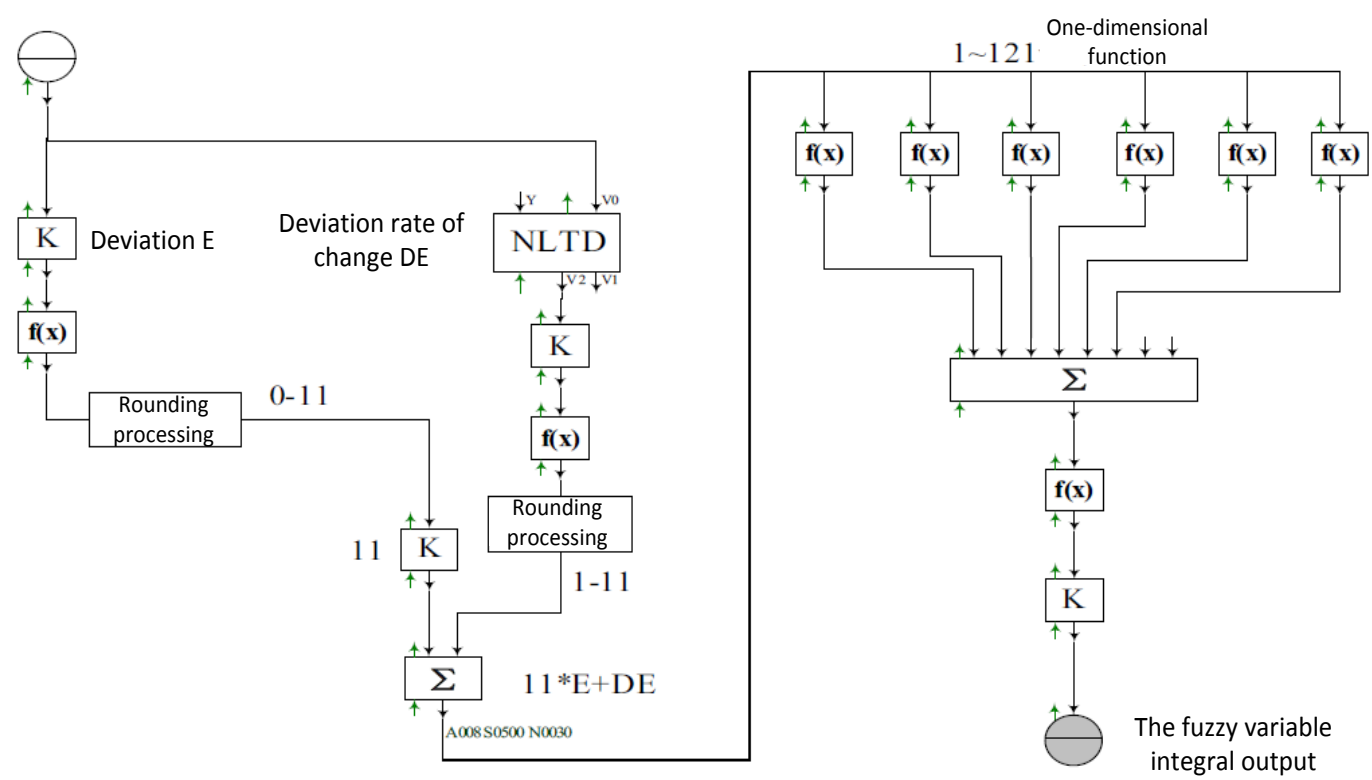

Fig. 4 Fuzzy control rule table

As shown in "Fig.4", implementation is the fuzzy control rule table $11 \times 11$, boiler regulator deviation E blurs and turn into $0 \sim 10$ after the integer dealing with, and deviation rate DE blurs and turn into $1 \sim 11$ after the integer dealing with, finally $11 \times 11$ two-dimensional table can be transferred into the required $1 \sim 121$ one dimension table according to the formula $11 \mathrm{x} E+\mathrm{DE}$. In practice, if you need other grades of two-dimensional table, can do according to this method.

\section{To optimize the application}

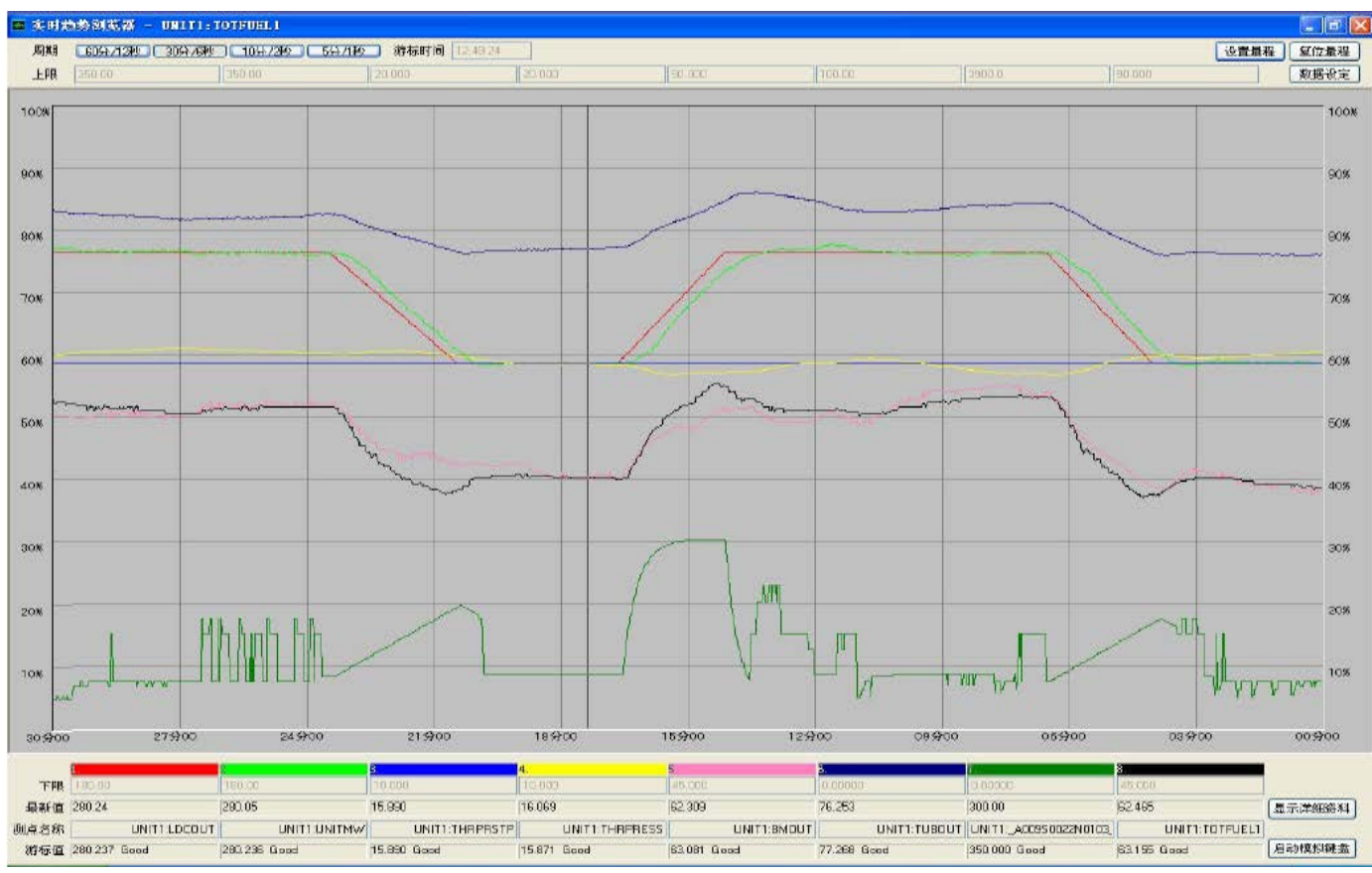

Fig. 5 Variable load test curves

To optimize the design of a Hangzhou company 350MW thermal power unit coordinated control system according to the control scheme, and make a load test of the range of 50MW, rate set of 12MW/Min after finishing the parameter setting , as shown in Fig. 5.Test data shows that on the basis of guaranteeing the stability of the unit parameters, output adjusting range increased by $60 \% \sim 100 \% \mathrm{MCR}$ to $50 \% \sim 100 \% \mathrm{MCR}$, lifting load rate rise by $6.60 \mathrm{MW} / \mathrm{min}$ to $9.83 \mathrm{MW} / \mathrm{Min}$, up $48.9 \%$. 


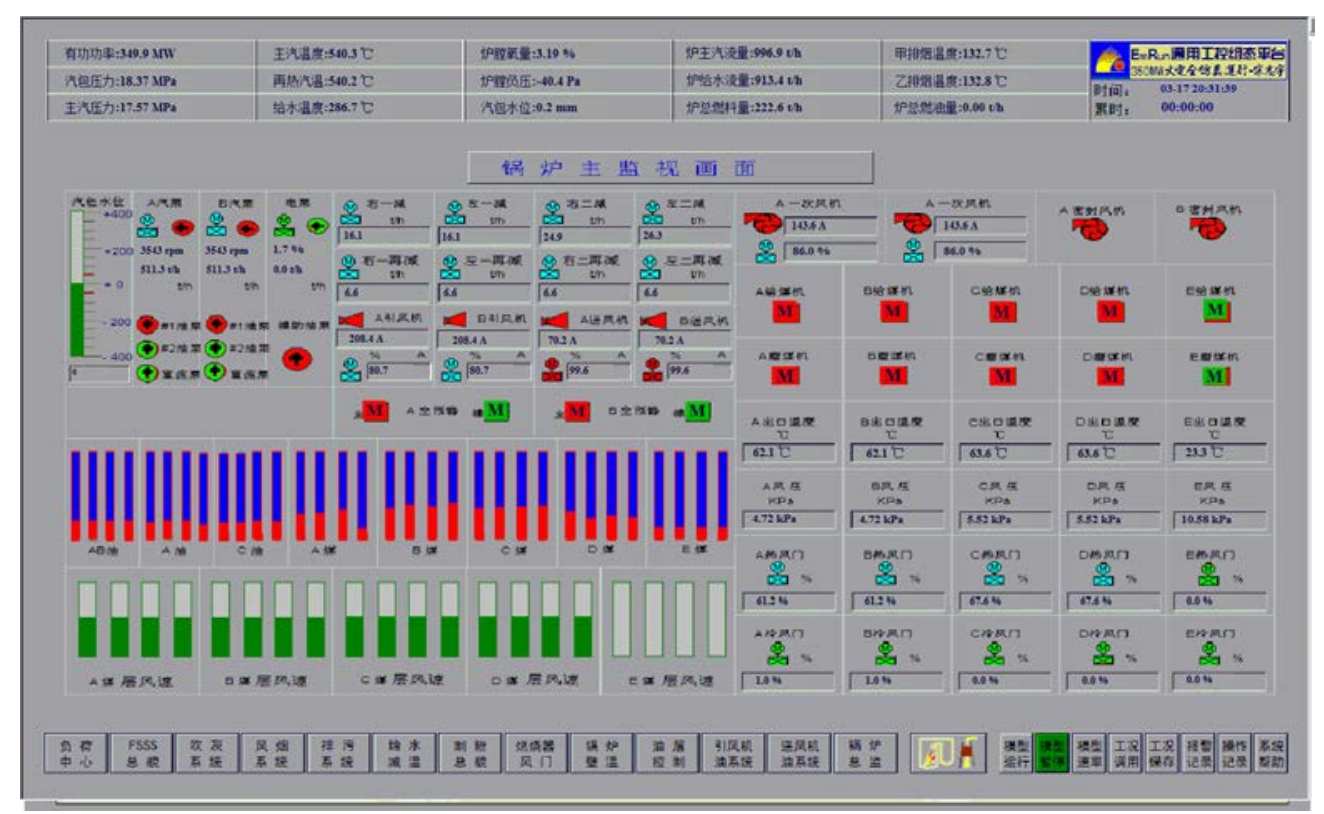

Fig. 6 Overall operating status of 350MW thermal power unit

\section{Conclusion}

In this paper, on the basis of direct energy balance method, the design of coordinated control strategy based on nonlinear control and fuzzy control improve the system control characteristic, effectively solve the questions, such as thermal power unit coordinated control machine, big inertia of furnace control model , nonlinear and strong coupling. After optimized design of 350MW thermal power unit coordinated control system according to this scheme, it significantly enhances power adjusting speed and the ability of severe peak load regulation and provide the technical support for improving the given ability of power grid to new energy power generation.

\section{Acknowledgments}

Financial support for this study was provided by Hangzhou science and technology projects, grant 20102011A23

\section{References}

[1] GUO Tao, GE Zhiping, ZHANG Shicai. Characteristic analysis of collaborative control system of 330 MW CFB units and its application [J].Electric Power Technology and Environmental Protection, 2012, 04:56-58.

[2] ZHAO Zhidan, CHENZhigang,WANG Xiaoyong. Application of direct energy balance in coordinated controlanditsoptimization [J].Thermal Power Generation, 2008,04:1-5.

[3] ZHANG Jian-sheng, ZENG De-liang, ZHAO Zheng, LIU Ji-zhen. Energy demand and Parameter adjustment of DEB400 coordinated control system [J]. Journal of North China Electric Power University, 2002, 04:60-64.

[4] Li Huijun. Optimal Research on Coordinated Control System of Home-made 300MW Unit[D].North China Electric Power University,2008.

[5] Zhang Jinzhe. Research on Optimized Strategy of Coordinate ControlSystem of 600MW Power Unit[D].North China Electric Power University,2012. 